\title{
Les Loranthaceae : un atout pour l'essor de la pharmacopée traditionnelle au Cameroun
}

\author{
Siegfried Didier DIBONG ${ }^{1 *}$, Nestor Laurier ENGONE OBIANG ${ }^{2}$, Ndongo DIN $^{1}$, \\ Richard Jules PRISO ${ }^{1}$, Victor TAFFOUO ${ }^{1}$, Henri FANKEM ${ }^{1}$, \\ Georges SALLE ${ }^{3}$ et AMOUGOU AKOA ${ }^{4}$ \\ ${ }^{1}$ Laboratoire d'Ecologie Végétale, Département de Biologie des Organismes végétaux, \\ Faculté des Sciences, B. P. 24157, Université de Douala, Cameroun. \\ ${ }^{2}$ Institut de Recherche d'Ecologie Tropicale (IRET), B. P. 13354 Libreville, Gabon. \\ ${ }^{3}$ Laboratoire de Parasitologie végétale, Université Pierre et Marie Curie (Paris VI), 4 place Jussieu \\ case courrier 155, 75252 Paris Cedex 05, France. \\ ${ }^{4}$ Département de Biologie et Physiologie Végétales, B. P. 812, Université de Yaoundé I, Cameroun. \\ *Auteur correspondent, E-mail: didierdibong@yahoo.fr
}

\section{RESUME}

La famille des Loranthaceae est largement distribuée dans les zones tropicales en Afrique, Amérique, Asie, Australie et s'étend en zones tempérées. Les Loranthaceae appartiennent à l'ordre des Santalales. Dans cette famille sont reconnus 950 espèces et 77 genres. Au Cameroun 26 espèces distribuées dans 7 genres sont citées. L'objectif de ce travail est d'évaluer, l'impact des Loranthaceae dans la pharmacopée traditionnelle des paysans. A travers un questionnaire sémi-structuré, 150 tradipraticiens en activité ont été interviewés en 2008 à Logbessou (quartier périphérique de la ville de Douala). Les réponses de l'enquête mise sur pied révèlent des informations selon lesquelles, les Loranthaceae sont des plantes parasites connues pour les dégâts considérables, occasionnés sur les essences ligneuses sauvages ou cultivées. Cependant, leur intérêt pour la pharmacopée traditionnelle est attesté. Les Loranthaceae fortifient le métabolisme et se présentent comme une panacée. Toutefois, l'ingestion des pseudobaies provoque des vomissements, de l'hypotension et des troubles nerveux. Les parties du végétal utilisées dans le traitement sont les feuilles, les rameaux et la tige sous forme d'extraits aqueux. Les allergies sévères sont rares. Le mode d'action des extraits aqueux des Loranthaceae européennes aux niveaux cellulaire et moléculaire est discuté.

() 2009 International Formulae Group. All rights reserved.

Keywords: Loranthaceae, plantes parasites, dégâts, pharmacopée traditionnelle.

\section{INTRODUCTION}

Les Loranthaceae sont des plantes hémiparasites épiphytes qui puisent l'eau et les sels minéraux dans les tissus de l'hôte, grâce à un système endophyte complexe (Sallé, 1978).

Le volet thérapeutique très développé en Europe depuis le dix-neuvième siècle est très recommandé dans la médication complémentaire de plusieurs cancers déclarés, particulièrement les cancers solides (Grossarth-Maticek et Ziegler, 2007).
L'intérêt porté aux Loranthaceae européennes par le monde scientifique et médical s'accroît lorsque Steiner en 1920, a mis l'accent sur une de leurs propriétés curatives. Cet auteur affirma, sans toutefois l'avoir démontré, que Viscum album L. (Loranthaceae) était un remède possible contre le cancer (Kienle et Kiene, 2007). Pourtant, en Afrique les études concernant les Loranthaceae sont orientées exclusivement vers la recherche des méthodes de lutte pour pallier aux baisses de rendements 
des récoltes qu'elles occasionnent (Dibong et al., 2008).

Le présent travail, une enquête menée en 2008 au quartier Logbessou située à la périphérie de la ville de Douala (point kilométrique 14) sur 150 tradipraticiens vise à pallier cette lacune.

\section{METHODOLOGIE}

\section{La région d'étude}

Logbessou est un quartier périphérique situé à 14 kilomètre au Nord-Est de la ville de Douala (Figure 1). Douala a pour coordonnées géographiques : latitude, $03^{\circ} 40^{\prime}-04^{\circ} 11^{\prime} \mathrm{N}$; longitude, $09^{\circ} 16-09^{\circ} 52^{\prime} \mathrm{E}$; altitude, $13 \mathrm{~m}$. Le climat appartient au domaine équatorial d'un type particulier dit « camerounien ». Il se caractérise par deux saisons avec une longue saison de pluies (au moins 9 mois), des précipitations abondantes (environ $4000 \mathrm{~mm}$ par an), des températures élevées $\left(26,7^{\circ} \mathrm{C}\right)$ et stables. La moyenne minimale de température à Douala pour 30 années (1961-1990) est de $22,6{ }^{\circ} \mathrm{C}$ en juillet et la moyenne maximale de température de $32,3{ }^{\circ} \mathrm{C}$ en février. L'humidité relative de l'air reste élevée toute l'année et avoisine $100 \%$ (Din et al., 2008).

\section{Enquête de connaissance des Loranthaceae}

La méthode est basée sur une enquête initiée auprès des personnes de sexe masculin, âgées de 30 à 60 ans environs ayant une bonne connaissance de la pharmacopée traditionnelle et s'exprimant couramment en français ou en anglais. Elles exercent dans l'informelle mais sont reconnues comme tradipraticiens au quartier Logbessou située à la périphérie de la ville de Douala (point kilométrique 14). L'enquête a été menée sur toute l'étendue de la banlieue d'avril à mai 2008, période durant laquelle les Loranthaceae sont en fleurs et/ou en fruits et donc faciles à identifier. L'interview est de type semi-structuré (Dahdouh-Guebas, 2000) avec un questionnaire préalablement élaboré comportant des questions fermées (à répondre par oui ou non) et des questions ouvertes (la latitude est laissée au répondant d'exprimer son point de vue).

Cent cinquante tradipraticiens en activité ont été interviewés en 2008. Les questions ont concerné la biologie, l'écologie, les méthodes de lutte et les vertus médicinales des Loranthaceae.

\section{RESULTATS}

Ecologie, biologie et méthodes de lutte contre les Loranthaceae par les tradipraticiens de Logbessou

Les tradipraticiens dans leur majorité (90\%) reconnaissent les Loranthaceae sur les arbres hôtes. Ce sont des plantes parasites des essences ligneuses sauvages ou cultivées, consommées ou ornementales connues sous plusieurs appellations : «cancer» en français, « samacopé » en anglais local, «tsap'la » en langue Menoua, «bara awali » en Béti. Seule l'existence d'une espèce est signalée par les paysans. L'espèce est reconnaissable à $78 \%$ par les paysans à partir de ses fleurs de couleur jaune avec l'apex rouge. Sa forme caractéristique est celle de bûchette d'allumette. Il s'agit de Phragmanthera capitata (Sprengel) S. Balle, répandue sur les essences ligneuses forestières, fruitières et ornementales.

Le cycle biologique des Loranthaceae reste peu connu des tradipraticiens. Des personnes interrogées, $58 \%$ parmi elles connaissent que les graines sont disséminées par les oiseaux ; $41 \%$ maîtrisent la période de fructification de ces plantes. La majorité des paysans (81\%) ignorent que les Loranthaceae sont d'origine forestière et $18 \%$ seulement appliquent la méthode curative de l'entaille de l'hôte et du parasite avec habilité.

Les inventaires floristiques des Loranthaceae dans les agroécosystèmes de Logbessou n'ont révélé que la présence de deux espèces (Figure 2): Phragmanthera capitata ubiquiste et Tapinanthus ogowensis spécialiste de Dacryodes edulis. Leurs fréquences relatives estimées sont respectivement de $98 \%$ et $2 \%$.

\section{La thérapeutique par les Loranthaceae}

Les Loranthaceae sont reconnues par les tradipraticiens de la région de Douala comme possédant un certain nombre d'usages thérapeutiques pour soigner ou contrôler une large gamme de maladies telles que les attaques des nerfs, le bourdonnement des oreilles, les convulsions, les crampes chroniques, les diabètes, les difficultés respiratoires, les douleurs dues au rhumatisme, l'épilepsie, l'étourdissement, la goutte, les hémorragies utérines, l'hypertension et l'hypotension, le mal de dos, le mal de reins, la ménopause, la migraine, 


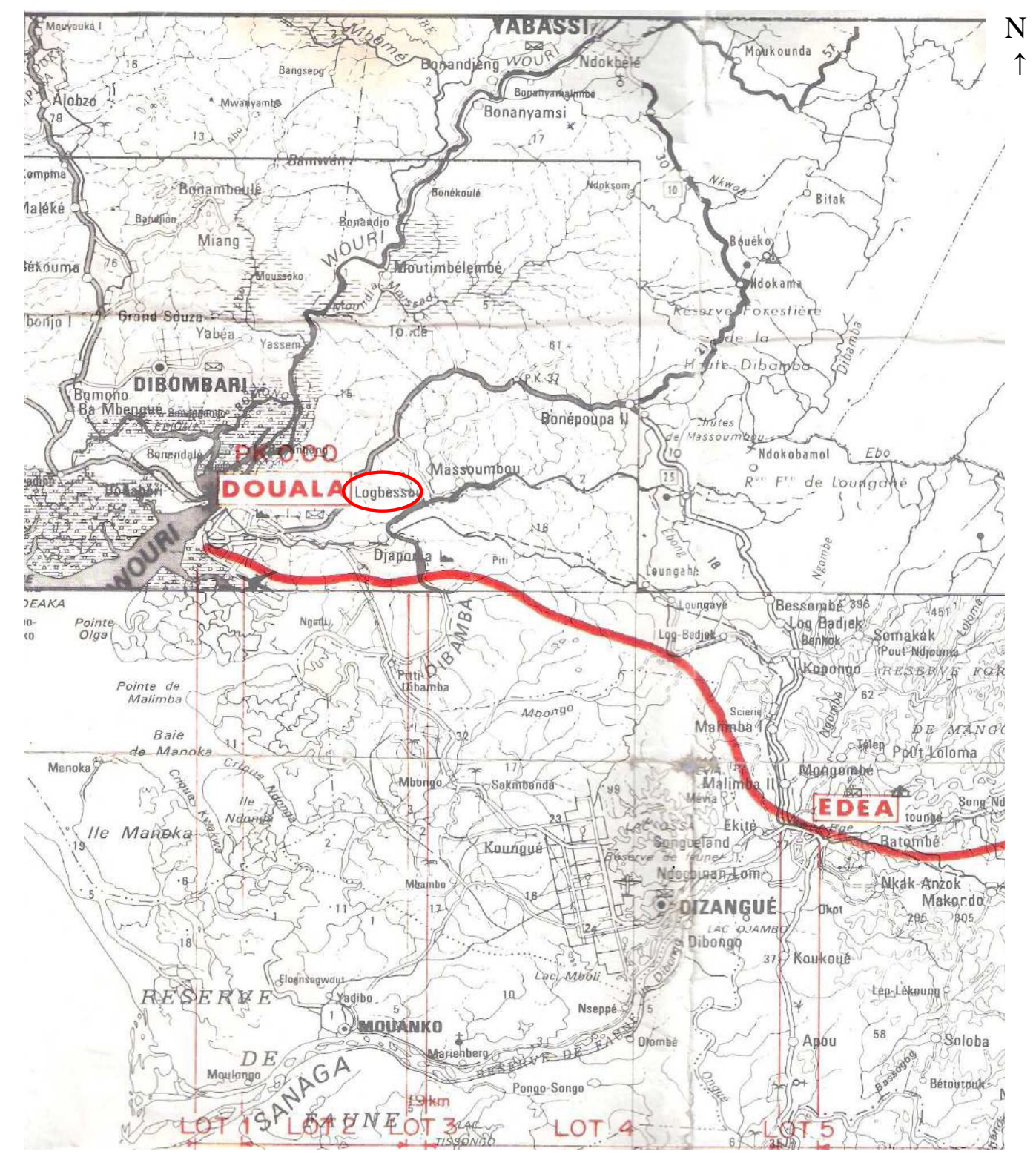

Zone d'étude

E : $1 / 500000$

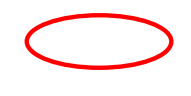

Figure 1: Localisation de la région de Douala.

les palpitations cardiaques, la purification générale, les troubles des règles, le saignement du nez. Les paysans de la tribu «banen» reconnaissent aussi chez les Loranthaceae, des pouvoirs mystiques servant à créer au sein d'un groupe de sorciers la divergence.

Les maladies citées par tous les tradipraticiens pour lesquelles les
Loranthaceae sont utilisées pour les traitements sont 22 au moins. Ces plantes parasites fortifient le métabolisme et se présentent comme une panacée pour les populations concernées. Selon ces tradipraticiens, les Loranthaceae sont utilisées depuis fort longtemps comme plantes médicinales en Afrique. 

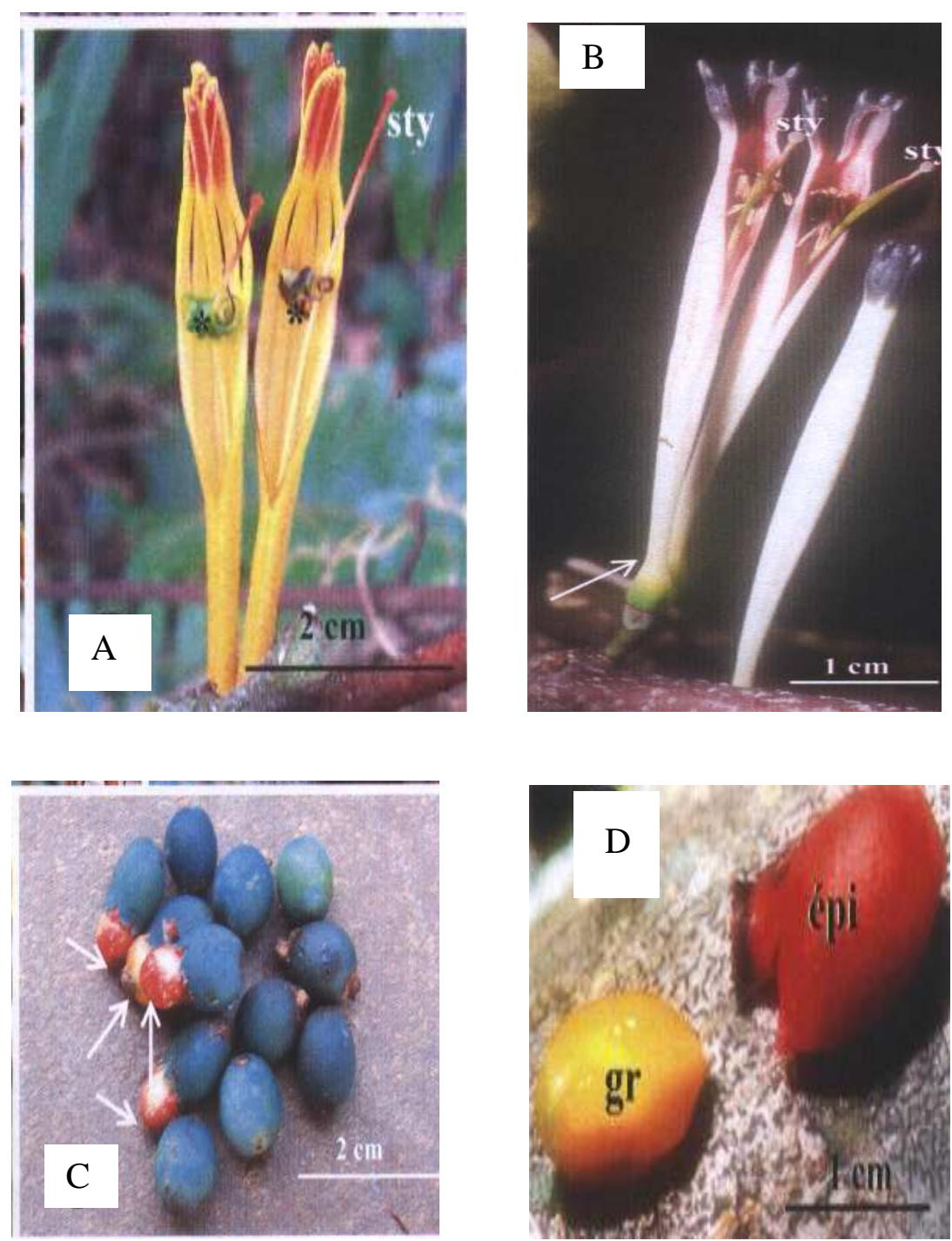

Figure 2 : A : Détail de deux fleurs épanouies de $P$. capitata, les étamines, ${ }^{*}$ s'enroulant sur ellesmêmes vers l'intérieur et recouvrent le style (sty) bicolore; B : Détail de deux fleurs épanouies de $T$. ogowensis, fortement étranglées à la base (flèche), montrant un style (sty) arrondi à son extrémité ; $\mathrm{C}$ : Baies adultes, renfermant une graine recouverte par une viscine orange ou rouge (flèches); D : graine (gr) entourée de viscine jaune et débarrassée de son épicarpe (épi). (Dibong et al., 2009a).

Les parties de la plante entière utilisées dans les divers traitements proposés sont les feuilles, les rameaux et la tige. Les Loranthaceae n'ont pas de racines. Ces dernières sont remplacées par le suçoir, véritable pont physiologique avec l'hôte. Les fruits sont des pseudobaies (Lemesle et Dupuy, 1971) et toxiques aux dires des tradipraticiens, lorsqu'elles sont avalées. L'ingestion des pseudobaies provoque des vomissements, de l'hypotension et des troubles nerveux. Pour un nombre de fruits supérieur à dix, la mort par arrêt cardiaque est inéluctable.

Les préparations utilisées sont les feuilles fraîches en infusion ou macérées. Cependant, la posologie reste aléatoire. De même, les modalités d'action des extraits de Loranthaceae sont mal maîtrisées et/ou inconnues des tradipraticiens. 


\section{DISCUSSION \\ Les propriétés pharmacodynamiques des Loranthaceae}

Les propriétés pharmacodynamiques pour lesquelles les tradipraticiens donnent des informations fiables, en accord avec la littérature concernent le cœur et le pancréas. L'influence des extraits de Loranthaceae sur le pancréas est telle qu'elles remédient aux causes du diabète, lorsque la cure est prolongée (Swanson-Flatt et al., 1989; Gray et Flatt, 1999 ; Onal et al., 2005 ; Orhan et al., 2005).

L'utilisation des Loranthaceae dans le traitement de l'hypotension a bien été démontrée. Selon Swanson-Flatt et al. (1989), Viscum album L. serait le seul hypotenseur végétal à action bien définie. Le même auteur préconise aussi son emploi contre l'artériosclérose, certains troubles nerveux, congestifs et de ménopause. Les tradipraticiens reconnaissent que les Loranthaceae interviennent dans la régulation des menstrues liées à des troubles du cycle : menstrues abondantes, hémorragies utérines, pertes de sang après accouchement, troubles de la ménopause (battement de cœur, palpitations, bouffées de chaleur, sensation d'angoisse et d'étouffement). Leur rôle antiinflammatoire est également attesté par les tradipraticiens: soulagement des douleurs du rhumatisme.

Karakas et al. (2008a) pensent que la viscotoxine serait responsable de l'action des extraits de Viscum album L. sur la circulation sanguine. Mais d'autres auteurs lui reconnaît des effets toxiques à faible ou forte dose (Rosell et Samuelsson, 1966 ; Lutomski, 1986). Néanmoins, Viscum album L. entre dans la composition d'une vingtaine de spécialités vendues en France sous forme de poudres ou d'extraits aqueux et il est surtout prescrit en médecine homéopathique (Baudino et Sallé, 1987). Karakas et al. (2008b) mentionnent que $V$. album L. subsp. album est un antispalmodique utilisé dans les cas de coqueluche, d'asthme et de certains types de convulsions. Il a également des propriétés antidiurétiques azoturiques et il est préconisé dans les néphrites à rétention azotée. Les tradipraticiens relèvent également les vertus des Loranthaceae dans le processus de vasodilation (Karakas et al., 2008b). La force régulatrice sur le système circulatoire est donc confirmée avec V. album en Europe (Ergun et al., 1995 ; Cook et Samman, 1996 ; Deliorman et al., 2000 ; Tenorio et al., 2005). Toutefois, cette propriété est infirmée par les résultats publiés par Radenkovic et al. (2006). Mais, ces résultats ne sont pas suffisants pour insinuer que les extraits de feuilles de $V$. album L. augmentent le tonus intestinal. Ces plantes sont aussi efficaces contre l'hypertension et l'hypotension artérielles. Leur utilisation est recommandée en cas d'artériosclérose du cerveau : étourdissements, vertiges, bourdonnement des oreilles, douleurs de la poitrine.

D'autres maladies mentionnées par les tradipraticiens montrent que les Loranthaceae ont des substances qui purifient le corps. D'après Gaultier (1938), à la fin du XVIe, Viscum album L. était aussi recommandé dans les cas d'épilepsie.

De nombreux travaux scientifiques ont montré la propriété cancérostatique de Viscum album L. (Mengs et al., 2002 ; Ernst et al., 2007 ; Harmsma et al., 2004 ; Urech et al., 2005 ; Grossarth-Maticek et Ziegler, 2008). Selon ces théories, le $V$. album L. tendrait à restaurer les «forces formatrices» de l'organisme menacé par les tendances prolifératrices des cellules. Ces préparations sont commercialisées dans le monde et particulièrement en Europe centrale sous le nom d'Abnobaviscum, Helixor, Iscador et de V. album fermenté (Kienle et Kiene, 2007). Le traitement du cancer par $V$. album L. a été introduit en 1920 par Steiner et Wegman, fondateurs de la médecine anthroposophique. Celle-ci recommande des formes injectables de $V$. album L. comme traitement pour le cancer surtout les tumeurs solides (Mahfouz et al., 1998 ; Matthes et al., 2005).

Les extraits de Viscum album L. sont préconisés dans trois principaux cas : tumeurs inopérables, prophylaxie des récidives et traitement de l'état précancéreux (Kienle et Kiene, 2007). Cependant, Matthes et al. (2005) pensent que l'efficacité de ce traitement reste controversée.

D'autres maladies traitées par les extraits de Viscum album L. ou «gui » européen n'ont pas été mentionnées par les tradipraticiens. Ce sont entre autres: les choliques intestinales (Karakas et al., 2008b), les hépatites (Harvey et Colin-Jones, 1981). 
Ainsi, des études supplémentaires et soutenues sont nécessaires pour élucider le mécanisme d'action des différents composés chimiques des Loranthaceae. La compréhension des propriétés curatives des Loranthaceae en dépend.

Les allergies sévères dues à la médication prolongée ou abusive sont rares (Bauer et al., 2005). Cependant, compte tenu des dosages standards et de l'interférence potentielle avec une nutrition normale, les parents doivent être prudents au sujet de leur emploi notamment pour les enfants. En effet, les pseudobaies et peut-être les feuilles peuvent causer une toxicité sévère (Spiller et al., 1996). Les femmes enceintes ou qui allaitent, les personnes ayant une hépatite sévère ou atteintes des maux de reins sont également exempts de cette médication. De plus, il semble que les Loranthaceae tropicales seraient plus toxiques que les Loranthaceae européennes (Spiller et al. ; 1996; Krenzelok et al., 1997). Des études complémentaires pour en avoir la certitude sont indispensables.

\section{Analyse des extraits de Loranthaceae}

Glatzel G (1983) montre que d'une façon générale, les Loranthaceae puisent dans leur hôte des quantités élevées de macroéléments minéraux tels que $\mathrm{Na}, \mathrm{K}, \mathrm{Ca}$ et $\mathrm{Mg}$. Les proportions relatives des différents ions dépendent de la nature des Loranthaceae et de l'hôte parasité. Les acides organiques et gras sont vingt et un environ et leur teneur varie considérablement avec la nature de l'arbre hôte (Baudino et Sallé, 1987). Cependant, les acides linoléique, palmitique et oléique représentent souvent plus de $80 \%$ de la fraction totale. Les glucides et substances apparentées appartiennent à trois groupes : les oses et polysaccharides, les polyols et l'acide ascorbique. Ainsi, les graines et feuilles de Loranthaceae contiennent plus de $7 \mathrm{mg}$ d'acide ascorbique par $\mathrm{g}$ de poids frais, ce qui est l'équivalent de la teneur de ce composé dans les citrons (Rikovski et Bessaritsh, 1966). Cette remarque n'est pas sans intérêt compte tenu des relations entre la vitamine C et le cancer (Sporn, 1980). Les composés phénoliques sont les acides phénoliques, les phénylpropanoïdes et les flavonoïdes. Selon Middleton (1996), les études sur les flavonoïdes sont assez complètes. Les composés phénoliques sont utilisés comme de puissants antioxydants (Kampa et al., 2000 ; Marfak, 2003). Les mécanismes d'action d'un antioxydant sont variés : piégeage direct des radicaux libres (atomes ou molécules contenant un électron non approprié) ou réaction stoechiométrique (réaction mole à mole avec les radicaux à éliminer); inhibition des enzymes et chélation des ions responsables de la production des radicaux libres; protection des systèmes de défense antioxydant (Halliwell, 1994).

L'équipement pigmentaire des Loranthaceae est celui des végétaux supérieurs et ne semble pas être influencé par la nature de l'hôte (Deliu et Stirban, 1976). Les saponosides, stéroïdes et terpènes ont été également identifiés (Obata, 1941). Les protéines et les substances associées sont : les acides aminés, amines et le viscumamide (peptide cyclique), les viscotoxines, protéines de Vester et lectines. Les trois dernières molécules qui ont en commun d'être des protéines semblent être largement responsables de l'action cancérostatique des extraits de Loranthaceae (Baudino et Sallé, 1987). Enfin les alcaloïdes et les substances de croissance sont également cités (Krivokapic, 1977).

La pharmacopée traditionnelle dans la médication pour le traitement des maladies est très pratiquée par la majorité des populations rurales car elle reste à la portée de toutes les bourses. Pour cela, cette pharmacopée doit être valorisée. Il n'est pas seulement important d'y associer des produits pharmaceutiques pour des raisons économiques mais aussi en raison d'une pénurie aigue de ces produits (Sofowora, 1984). Des travaux doivent être conduits pour déterminer les constituants chimiques actifs des extraits aqueux de Phragmanthera capitata, angiosperme ubiquiste dont le parasitisme correspond à toutes les variations écologiques du pays (Balle, 1982). Dans la région de Douala, le pourcentage de parasitisme de cette Loranthaceae est de $76,14 \%$ ( $<<0,05$ ) (Dibong et al., 2009b). Les résultats probants de ces travaux permettraient le conditionnement industriel des extraits $P$. capitata et la réduction sensible des pertes de rendement souvent considérables pour les arbres fruitiers cultivés parasités (Sonké et al., 2000; Engone et al., 2005, 2006). 
La lutte contre Phragmanthera capitata, abondante et virulente dans la région de Douala (Dibong et al., 2009b) ne doit plus consister à la suppression manuelle des touffes telle que le préconisent certains travaux (Dibong et al., 2008). Il faudrait plutôt envisager son usage dans la médecine moderne pour le traitement du cancer et des pathologies impliquées comme c'est le cas avec Viscum album L. en Europe.

\section{Conclusion}

L'étude réalisée avec l'apport des tradipraticiens de Logbessou (point kilométrique de Douala) permet de constater que des lacunes subsistent concernant la phénologie, l'écophysiologie et la biologie des Loranthaceae. Cependant, l'apport des Loranthaceae dans la pharmacopée traditionnelle est indéniable. Leur éradication préconisée par la méthode de lutte mécanique ne doit plus être envisagée par les populations concernées. Il faut introduire la notion de lutte intégrée en maintenant les Loranthaceae à un seuil acceptable sur l'hôte et dans l'environnement. Après destruction de la forêt, il faut veiller à ce que les hôtes potentiels maintenus dans les champs ne soient pas parasités. Il serait aussi judicieux, pour réduire le degré de parasitisme, d'introduire des hôtes pièges tel que le manguier particulièrement résistant aux Loranthaceae. Le safoutier, spécialiste de Tapinanthus ogowensis dans la région littorale, dans une certaine mesure, jouerait ce rôle lorsque Phragmanthera capitata est présente dans le milieu.

A l'heure actuelle, les modalités d'action des extraits de Loranthaceae sont loin d'être connues. Cependant, il est certain qu'il agit chez l'homme en stimulant les systèmes de défense de l'organisme, sans que l'on puisse préciser comment. Une action directe ou indirecte au niveau des acides nucléiques est fortement soupçonnée, sans que l'on puisse trancher entre trois substances: protéines de vester, lectines ou viscotoxines. Il est très probable que ces deux derniers groupes de molécules soient responsables d'une très grande partie de l'activité des extraits de Viscum album L.

Pendant que les Européens s'engagent aujourd'hui dans la culture in vitro du Viscum album L. (Baudino et Sallé, 1987) pour pallier l'épuisement de ce médicament (appelé par abus parasite), des équipes de recherche sur cette famille doivent être mises sur pied en Afrique. Les axes de recherche devraient avoir deux volets: isolement des extraits de Loranthaceae et dosage des protéines de Vester et viscotoxines, puis injections de ces substances purifiées sur différentes lignées cellulaires pour suivre leur action.

Les Loranthaceae constituent à n'en plus douter une richesse indéniable. Car les extraits purifiés varient suivant le couple hôte/parasite. Pour le Cameroun où il est reconnu 26 espèces de Loranthaceae réparties en 7 genres (Balle, 1982) et pour autant d'hôtes de $P$. capitata, de telles recherches sont indispensables. De même, les études des interactions hôtes/Loranthaceae devraient être perpétuées compte tenu des corrélations spécifiques mises en jeu.

\section{REFERENCES}

Balle S. 1982. Loranthacées. In Flore $d u$ Cameroun (vol. 23), Satabié B, Leroy JF (eds). Délégation Générale à la Recherche Scientifique et Technique: Yaoundé, Cameroun ; 82p.

Baudino S, Sallé G. 1987. Les substances actives du gui. Propriétés pharmacologiques et applications thérapeutiques. Annales des Sciences Naturelles, Botanique, 13(8): 45-72.

Bauer C, Oppel T, Rueff F. 2005. Anaphylaxis to viscotoxins of mistletoe (Viscum album) extracts. Ann. Allergy Asthma. Immunol., 94: 86-89.

Cook NC, Samman S. 1996. Flavonoids chemistry metabolism, cardioprotective effect, and dietary sources. Nutr. Biochem., 7: 66-76.

Dahdouh-Guebas F, Mathenge C, Kairo JG, Koedam N. 2000. Utilisation of mangrove wood products around Mida creek (Kenya) among subsistence and commercial users. Economic Botany, 54(4): 513-527.

Deliorman D, Calis I, Ergun F. 2000. Studies on the vascular effects of the fractions and phenolic compounds isolated from Viscum album ssp. album. J. Ethnopharmacol., 72: 323-329.

Deliu C, Stirban M. 1976. Annual dynamics of assimilatory pigments in $V$. album and its host plant, Populus tremula L. 
Contrib. Bot., Gradina. Bot. Univ. BabesBolyai Cluj., 243-249.

Dibong SD, Din N, Priso RJ, Taffouo VD, Fankem H, Sallé G, Amougou A. 2008. Parasitism of host trees by the Loranthaceae in the region of Douala (Cameroon). African Journal of Environmental Science and Technology, 2(11): 22-30.

Dibong SD, Engone Obiang NL, Din N, Priso RJ, Taffouo VD, Fankem H, Sallé G, Amougou A. 2009a. Artificial infestations of Tapinanthus ogowensis (Engler) Danser (Loranthaceae) on three host species in the Logbessou Plateau ( Douala, Cameroon). Afr. J. Biotechnol., 8(6): 1044-1051.

Dibong SD, Engone Obiang NL, Din N, Priso RJ, Taffouo VD, Fankem H, Sallé G, Amougou A. 2009b. Niveau d'infestation des arbres fruitiers des groupements végétaux par Phragmanthera capitata (Sprengel) S. Balle (Loranthaceae) dans la région littorale du Cameroun. Int. J. Biol. Chem. Sci., 3(2): 347-354.

Din N, Priso JR, Dibong SD, Amougou A. 2008. Logging activities in mangrove forests: A case study of Douala Cameroon. African Journal of Environmental Science and Technology, 2(2): 22-30.

Engone Obiang NL, Paré J, Duredon J, Sallé G. 2005. Germination et développement de la plantule de Helixanthera mannii (Oliv.) Danser (Loranthaceae) sur cacaoyer (Theobroma cacao L.) au Gabon. Revue de Cytologie et Biologie Végétales- Le Botaniste, 29:13-21.

Engone Obiang NL, Sallé G. 2006. Faut-il éradiquer Phragmanthera capitata, parasite des hévéas en Afrique? C.R. Biologies, 329: 185-195.

Ergun F, Deliorman D, Akar F. 1995. Screening of various Viscum album L. Samples of their possible vascular effects. Guede. J. Fac. Pharm. Gazi., 12: 153158.

Ersnt E, Schmidt K, Steuer-Vogt MK. 2007. Mistletoe for cancer? Int. J. Cancer, 107: 262-267.

Gaultier R. 1938. Le gui de chêne. Aesculape, 28: 89-94.

Glatzel G. 1983. Mineral nutrition and water relations of hemiparasitic Mistletoes: a question of partitioning. Experiments with Loranthus europaeus on Quercus petraea and Quercus robur. Oecologia, 56: 193-201.

Gray AM, Flatt PR. 1999. Insulin-secreting activity of the traditional antidiabetic plant Viscum album (mistletoe). J. Endocrinol., 160: 409-414.

Grossarth-Maticek R, Ziegler R. 2007. Prospective controlled cohort studies on long-term therapy of cervical cancer patients with a mistletoe preparation (Iscador). Forsch Komplementarmed, 14: 140-147.

Grossarth-Maticek R, Ziegler R. 2008. Randomized and non randomized prospective controlled cohort studies in matched pair design for the long term therapy of Corpus uteri cancer patients with a mistletoe preparation (Iscador). Eur. J. Med. Res., 13: 107-120.

Halliwell BJ. 1994. Free radicals as mediators of tissue injury and disease. Crit. Rev. Toxicol., 23: 21-48.

Harmsma M, Gromme M, Ummelen M. 2004. Differential effects of Viscum album extracts Iscador $\mathrm{Qu}$ on cell cycle progression and apoptosis in cancer cells. Int. J. Oncol., 25: 1521-1529.

Harvey J, Colin-Jones DG. 1981. Mistletoe hepatitis. Br. Med. J. (clin. Res. Ed), 282: 186-187.

Kampa M, Hatzoglou A, Notas G, Damianaki A, Bakogeorgou E, Gemetzi C, Kouroumalis E, Martin PM, Castanas E. 2000. Wine antioxidant polyphenols inhibit the proliferation of human prostate cancer cells lines. Nutr. Cancer, 37: 223233.

Karakas A, Turker AU, Gunduz B. 2008a. Effect of European Mistletoe (Viscum album L. Subsp. album) extracts on activity rhythms of the syrian hamsters (Mesocricetus auratus). Nat. Prod. Res., 22: 996- 1006.

Karakas A, Serin E, Gunduz B, Turker AU. 2008b. The effects of Mistletoe (Viscum album L. subsp. Album) extracts on isolated intestinal contractions. Turk. J. Biol., 32:237-242.

Kienle GS, Kiene H. 2007. Complementary cancer therapy: a systematic review of propective clinical trials on 
anthroposophic Mistletoe extracts. Eur. J. Med. Res., 12: 103-119.

Krenzelok EP, Jacobsen TD, Aronis J. 1997. A mistletoe exposures. AM. J. Emerg. Med., 15: 516-520.

Krivokapic C. 1977. Plant growth substances in Viscum album L. Proc. Int. Symp. Plant Growth Regulators, Sofia, 286-294.

Lemesle R, Dupuy P. 1971. Le gui (Viscum album). Morphologie, biologie, propriétés. Quart. J. Crude Drug Res., 11: 1721-1726.

Lutomski J. 1986. Evaluation of the hypotensional activity of some mistletoe (Viscum album L.) drugs. Int. Meeting on Viscum album, Heidelberg.

Mahfouz MM, Ghaleb HA, Zawawy A, Scheffler A. 1998. Signifiant tumor reduction, improvement of pain and quality of life and normalization of sleeping patterns of cancer patients treated with a high dose of mistletoe. Ann. Oncol., 9(2): 129-138.

Marfak A. 2003. Radiolyse gamma des flavonïdes. Etude de leur activité avec les radicaux issus des alcools : formation des depsides. Thèse de Doctorat de la Faculté de Pharmacie de Limoges, 220p.

Matthes H, Schad F, Buchward D, Schenk G. 2005. Endoscopic ultrasound-guided fine needle injection of Viscum album L. (mistletoe; Helixor M) in the therapy of primary inoperable pancreas cancer: a pilot study. Gastroenterology, 128(4 suppl. 2): 433, T988.

Mengs U, Gothel D, Leng-Peschlow E. 2002. Mistletoe extracts standardized to mistletoe lectins in oncology: review on current status of preclinical research. Anticancer Res., 22: 1399-1405.

Middleton EJr. 1996. Biological properties of plant flavonoids. An overview. Int. J. Pharmacogn., 34: 344-348.

Obata Y.1941. The components of Viscum album III. The unsaponifiable matter of the leaves, the so-called \&-viscol and \&viscol. J. Agr. Chem. Soc. Japan, 17: 784-786.
Onal S, Timur S, Okutucu B. 2005. Inhibition of alpha-glucosidase by aqueous extracts of some potent antidiabetic medicinal herbs. Prep. Biochem. Biotechnol., 35: 29-36.

Orhan DD, Aslan M, Sendogdu N. 2005. Evaluation of the hypoglycemic effect and antioxidant activity of three Viscum album subspecies (European mistletoe) in streptozotocin-diabetic rats. J. Ethnopharmacol., 98: 95-102.

Radenkovic M, Ivetic V, Popovic M. 2006. Neurophysiological effects of mistletoe (Viscum album L.) on isolated rat intestines. Phytother. Res., 20: 374-377.

Rosell S, Samuelsson G. 1966. Effect of mistletoe viscotoxin and phoratoxin on blood circulation. Toxicon, 4: 107-110.

Sallé G. 1978. Origin and early growth of the sinkers of Viscum album L. Protoplasma 96: 267-273.

Spiller HA, Willias DB, Gorman SE, Sanftleban J. 1996. Retrospective study of mistletoe ingestion. Clin. Toxicol., 34: 405-408.

Sporn MB. 1980. Combination chemoprevention of cancer. Nature, 287: 107.

Sofowora A. 1984. Medicinal Plants and Traditional Medicine Published in Association with Spectrum. Books Ltd. Ibadan by John Wiley and Sons; 142-143.

Sonké B, Kenfack D, Tindo M. 2000. Parasitisme de l'avocatier (Persea americana, Lauraceae) par les Loranthacées de la région de Yaoundé (Cameroun). Fruits, 55: 325-331.

Swanson-Flatt SK, Day C, Bailey CJ, Flatt PR. 1989. Evaluation of traditional plant treatments for diabetes: studies in streptozotocin diabetic mice. Acta Diabetologica Latina, 26: 51-55.

Tenario FA, del Valle L, Gonzalez A. 2005. Vasodilator activity of the aqueous extract of Viscum album. Fitoterapia, 76: 204-209.

Urech K, Scher JM, Hostanska K. 2005. Apoptosis inducing activity of viscin, a lipophilic extract from Viscum album L. J. Pharm. Pharmacol., 57: 101-109. 